Pecvnia, 6 (2008), pp. 107-129

\title{
Tendencia de la responsabilidad social empresarial a la excelencia corporativa
}

Recibido: Febrero 2008 Aceptado: Junio 2008

\author{
Ma Ángela Jiménez Montañés \\ Angela.Jimenez@uclm.es \\ Universidad de Castilla La Mancha \\ Economía Financiera y Contabilidad \\ Fac. de Ciencias Jurídicas y Sociales \\ Cobertizo de San Pedro Mártir, s/n \\ 45071 Toledo (España)
}

En el entorno económico actual es necesario, para asegurarse el éxito y una posición competitiva, no sólo un control de costes, sino también innovación, flexibilidad, calidad, distribución de productos en un mercado internacional. En especial, deben considerarse tres tipos de aspectos: los referentes a la influencia de la estrategia y de la estructura organizativa en el diseño de la política de gestión, donde enmarcamos la gestión de la calidad total, la elaboración del presupuesto y el cálculo de las desviaciones y la influencia de las personas, la cultura y el entorno de una empresa.

La aplicación y el análisis de las actividades para la Gestión continua de la calidad supone un estilo nuevo de dirección, basado en unos principios diferentes a los considerados en el estilo tradicional, y centrados en las ideas
In the present economic environment, it is necessary, in order to ensure the success and a competitive position, not only a cost control, but also product innovation, flexibility, quality and distribution in an international market. Three kinds of aspects must be considered: the referring ones to the influence of the strategy, the organizational structure in the design of the management policy, where we frame the management of the total quality, the elaboration of the budget and the calculation of the deviations and the influence of the company's people, culture and environment.

The application and the analysis of the activities for the continuous management of the quality implies a new management style based on principles different from the considered ones in the traditional style, and focused on the 
fundamentales del "Gobierno Corporativo". La aplicación del estudio nos permitirá contestarnos a cuatro preguntas básicas, que nos servirán como autocontrol en la gestión desarrollada. Estas cuestiones son ¿Qué cultura empresarial seguimos y quienes somos?, ¿Qué actuaciones estamos desarrollando en este entorno de excelencia?, ¿Conocemos a nuestros clientes y sus demandas, presentes y futuras? ¿La política seguida con los proveedores es la acertada en la generación de valor global? ¿Se han cumplido los objetivos establecidos y planificados? ¿Cual es el camino a seguir para el futuro?.

Palabras clave: Calidad, Excelencia, Responsabilidad social. fundamental ideas of the "Corporate Governance". The application of the study will allow us to answer four basic questions, that will serve as an automatic control in the developed management. These questions are: What entrepreneurial culture are we following and who are we?, What performances are we developing in this environment of excellence?, Do we know our customers and their future and presents demands? The policy we have followed with the suppliers is the right one in the generating global value? Have the established and planned objectives been fulfilled? What is the way to be followed in the future?.

Key words: Quality, excellence, Social Responsibility. SOCIAL EMPRESARIAL

1. MARCO GENERAL DE DESARROLLO: LA RESPONSABILIDAD

La Responsabilidad Social Empresarial (RSE), de marcada actualidad en los momentos actuales se define de múltiples formas, atendiendo a los diferentes ámbitos, entornos y campos donde se aplica. En términos generales, por RSE podemos considerar que se trata de la implantación voluntaria de sistemas de gestión, medición e información del comportamiento socialmente responsable de una organización, considerando los grupos de interés (stakeholders). Por lo tanto, se trata de un enfoque de negocio que pretende crear valor a l/p para los accionistas, asumiendo riesgos y oportunidades de aspectos económicos, sociales y medioambientales y, en definitiva, se centra en una contribución al desarrollo sostenible.

Considerando las ideas de PriceWaterhouseCoopers, como ideas de carácter general, la RSE puede establecerse como el desarrollo de estrategias de Sostenibilidad Corporativa, centradas en los siguientes puntos:

- Integración voluntaria de preocupaciones sociales y medioambientales en las operaciones comerciales y relación con stakeholders.

- Compromiso continuo de actuación ética, contribuyendo al desarrollo económico y mejora de la calidad de vida de los trabajadores en la sociedad, en general. 
- Prácticas abiertas y transparentes en los negocios, basados en valores éticos y respeto por todas las partes interesadas.

En definitiva, se trata de una nueva dimensión empresarial preocupada por el concepto de sostenibilidad en tres dimensiones: económica, social y medioambiental, cuyo principal objetivo es el desarrollo sostenible, como vía de implantación de un modelo de empresa socialmente responsable, afectando a la empresa como sistema: gobierno, dirección estratégica, gestión y control, información, evaluación y validación externa, certificación, inversión, reconocimiento, incentivos de mercado.

El impacto de la RSC en la organización se desarrolla en tres dimensiones: dimensión económica, dimensión social y dimensión medioambiental. Pero en todo caso, el gran objetivo principal se concreta en el desarrollo sostenible y generación de valor. Para ello, es necesario establecer el desarrollo del sistema de gestión en tres dimensiones, centradas en resultados económicos, rentabilidad y rendimiento.

Dentro del concepto RSE se integran conceptos, dimensiones y objetivos, que constituyen los elementos conceptuales de la RSC. Entre ellos podemos destacar: responsabilidad, social, corporación, compromiso voluntario, organización, sociedad, acción social, auditoria social, capital relacional-capital intelectual y códigos de conducta. También se consideran los siguientes elementos: código de buen gobierno, desarrollo sostenible, empresa ciudadana, ética empresarial, filantropía estratégica, gestión medioambiental, marketing con causa social, reputación corporativa, sostenibilidad y triple cuenta de resultados, estados financieros sostenibles, responsables y éticos.

Si bien, se presenta un conjunto de limitaciones o dificultades, a la hora de su integración dentro de la filosofía empresarial. La más relevante podría ser la dificultad de implantación, considerando la integración de necesidades y expectativas de los grupos de interés en el proceso de toma de decisiones para la consecución del objetivo general que contribuya al desarrollo y beneficio mutuo de la organización y de los grupos de interés. Esto contribuye a definir una necesidad centrada en el establecimiento de mecanismos de control interno, en las tres dimensiones, comentadas anteriormente, que garanticen y faciliten las actividades internas y la coherencia con los fines de la empresas y las exigencias del entorno. Por lo tanto se requiere un adecuado control de gestión sostenible. 
La gestión empresarial responsable va a plantear tres grandes grupos de problemas:

- Problemas de identidad y de cultura empresarial. Evaluar permanentemente la situación del entorno en las vertientes socioeconómica-institucional y de mercado, para elaborar escenarios de referencia.

- Problemas del entorno, de posicionamiento y del compromiso con el exterior. Tipología de productos y precios y análisis de las situaciones de competencia, considerando las necesidades de la sociedad y la demanda del mercado.

- Problemas de instrumentación, de fijación de objetivos y de desarrollo de la actividad corporativa. Fijación de objetivos operativos y estratégicos. Económicos, sociales y medioambientales.

A su vez, estos grupos de problemas se identifican con tres grandes bloques de decisión empresarial, como son:

- La filosofía empresarial, identidad, instrumentación y entorno.

- Definición de políticas requeridas, centradas en la financiación, administración, posicionamiento y compromiso, tipología de productos, precios y competencia.

- Definición real y clara de los objetivos a alcanzar, dentro de la filosofía del Desarrollo sostenible y la generación de valor.

De manera gráfica, si interrelacionamos los tres grandes grupos de problemas con los tres grandes bloques de decisión empresarial, podemos deducir, que es la propia interrelación la única encargada de garantizar el cumplimiento de los objetivos.

Estos objetivos de la RSC pueden suministrar elementos de dirección y gestión para:

- Desarrollo de modelo de empresa ciudadana y desarrollo sostenible.

- Innovación y mejora de procesos de dirección, gestión, medición e información de las organizaciones. Obtención de la triple dimensión de la RSC. Satisfacción de necesidades de los grupos de interés.

- Generación de externalidades socialmente responsables. 
Figura 1: Interrelación de problemas y decisiones

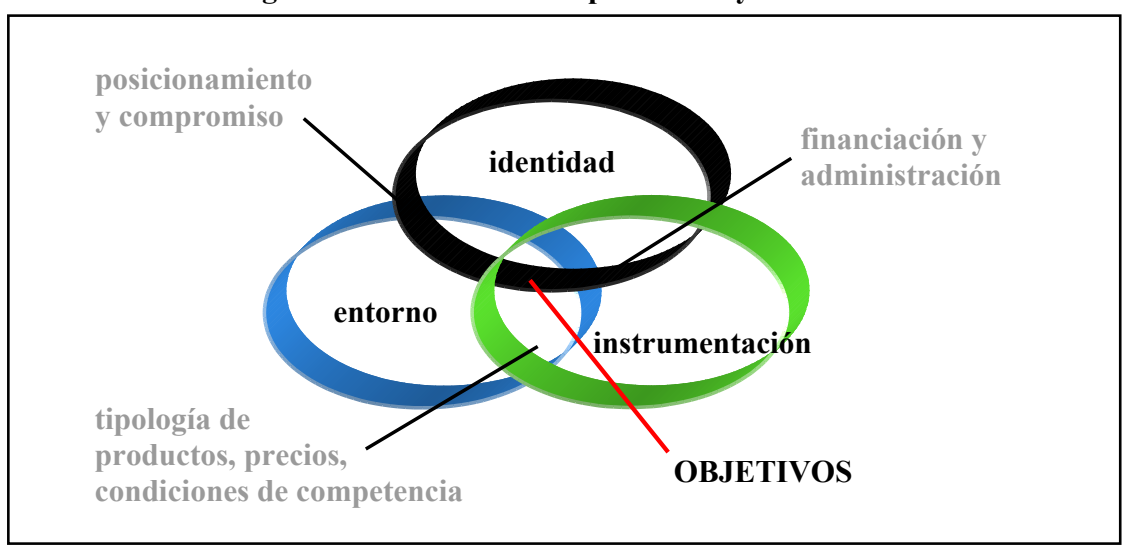

Para garantizar el cumplimiento de los objetivos de la RSC debemos definir una serie de variables, que se dividen en tres grandes bloques: variables clave, variables organizativas y variables genéricas. Dentro de las primeras se incluyen aquellas que constituyen los aspectos de los que depende la posición competitiva sostenible, entre los que podemos destacar:

- Coste de las actividades ( $A B C, A B M)$

- Coste de los productos y servicios

- Crecimiento de las ventas

- Seguridad en la función de aprovisionamiento (JIT, MRP, ERP, etc.)

- Eficiencia en inversiones

- Nivel de endeudamiento

- Tipo de interés de la financiación

- Cuota de mercado

- Capacidad de innovación

- Flexibilidad del proceso productivo

- Plazo de entrega del producto final

- Calidad del servicio y del producto (TQM; ISO 9000:2000)

- Imagen pública

- Cotización en Bolsa y transparencia de información

- Motivación del personal

- Productividad

- Nuevas Tecnologías

- Acceso a la información

- Derecho a la información 
En relación al segundo bloque de variables se referencian las políticas de precios y de producción. Y por último, con relación a las variables genéricas, destacamos las del entorno, la competencia y la clientela.

Es dentro del primer grupo de variables donde enmarcamos el trabajo presentado, centrado en la valoración y análisis de la actividad de la calidad, como elemento integrante del nuevo sistema de gestión responsable.

2. LA GESTIÓN EMPRESARIAL RESPONSABLE: ADMINISTRACIÓN DE LA EXCELENCIA Y LA CALIDAD

Considerando el gráfico anterior, la fijación de los objetivos y la toma de decisiones, en relación al grado y momento de su consecución, dan lugar a las actividades de dirección y gestión empresarial, las cuales se sustentan en un conjunto de datos, es decir, en un sistema de información, constituido por el Sistema Informativo Contable. De ahí, se establece la triple relación entre el Sistema de Información Contable, las organizaciones y la consecución de la competitividad y la responsabilidad social y empresarial, como objetivo prioritario a alcanzar.

El objetivo de calidad es muy importante, puesto que para mantenerse $o$ introducirse en un sector o mercado, la calidad se constituye como el factor primordial. En este caso, el término calidad no puede relegarse únicamente, a la calidad de los productos, sino a un sentido más amplio de calidad integral de la empresa, tanto a nivel humano como material. Este nuevo sentido, supone un incremento en los costes empresariales, lo cual implica la aplicación de una gestión estratégica adecuada en el campo de los costes con un posicionamiento estratégico específico ligado a la cadena de valor y mediante el proceso adecuado de identificación de conductores o más propiamente drivers, que conduzcan a la reducción de los costes empresariales.

Para asegurar una posición competitiva, como variable socialmente responsable, es necesario, no sólo un control de costes, sino también innovación, flexibilidad, calidad, distribución de productos en un mercado internacional, etc. En esta situación, es difícil cuantificar en términos monetarios los objetivos, el resultado a priori de las decisiones y acciones que se tomen y el resultado final. 
En especial, deben considerarse tres tipos de aspectos:

- Los referentes a la influencia de la estrategia y de la estructura organizativa en el diseño de la política de gestión, donde enmarcamos la gestión de la calidad total.

- La elaboración del presupuesto y al cálculo de las desviaciones.

- Los que se refieren a la influencia de las personas, la cultura y el entorno de una empresa.

En el planteamiento de la dirección de las empresas, es donde se integra la gestión de la calidad total y de la excelencia, y por tanto, la gestión estratégica de los costes de calidad. Al mismo tiempo, para alcanzar la excelencia, dentro del proceso de mejora tendente a la calidad total, podemos identificar tres elementos básicos:

- Análisis de las actividades de la calidad, donde centramos el trabajo

- Análisis de los procesos de gestión

- Análisis y resolución de problemas y desviaciones

Si bien, no podemos decir que exista una definición estándar del concepto de estrategia, podemos establecer, en palabras de Porter (1999: 51) que la estrategia competitiva consiste en ser diferente y significa elegir deliberadamente un conjunto de actividades diferentes para prestar una combinación única de valor. Se puede hablar del concepto de estrategias genéricas, de liderazgo en costes, de diferenciación y de enfoque, para representar las posiciones estratégicas alternativas que se pueden presentar en un sector.

Tanto la eficacia operativa como la estrategia se comprenden mejor si se divide a la empresa en actividades, en los distintos procesos económicos que lleva a cabo para competir en cualquier campo. El análisis de actividades presenta una definición más restrictiva que la de las funciones tradicionales, porque nos permite definir una estructura conceptual para examinar sistemáticamente las actividades y la conexión con la ventaja competitiva que supone el análisis de la cadena de valor.

Dentro de la clasificación de actividades que se desarrolla en el modelo $A B C$, aquellas encaminadas al proceso continuo de apoyo al cliente se pueden relacionar con la política de la Calidad Total, centradas 
en la realización de todas las actividades de la forma más eficiente y bajo la filosofía de la mejor continua, destinadas a garantizar la calidad global de los productos para que respondan a las necesidades y demandas de los clientes, logrando una fuerte ventaja competitiva frente a sus más rivales competidores.

El concepto de competitividad puede relacionarse con la obtención de los niveles máximos de eficiencia y eficacia. La eficiencia se refiere a la economía de medios para acometer unos determinados fines y objetivos. Esta puede ser económica o técnica. La económica nos relaciona el producto por unidad de coste de los recursos utilizados, en contraposición con la eficiencia técnica que mide la producción de energía empleada. La eficiencia económica se refiere al coste y al valor.

En este entorno es donde vamos a analizar los diferentes conductores o drivers que puede utilizar la unidad competitiva para poder alcanzar una adecuada eficiencia económica en el campo de los costes de calidad, analizando su variabilidad atendiendo a la teoría tradicional de los costes y los diferentes conductores que podemos encontrar en un entorno estratégico.

La gestión estratégica de los costes enfoca como primer objetivo organizar la información para que la organización empresarial se mantenga competitiva, logrando la mejora continua de los productos y servicios de alta calidad que satisfacen a los clientes y consumidores al menor precio. Esto se logra mediante el diseño y adaptación continua de una combinación productiva global que mantenga el valor percibido por el cliente (Mallo y Jiménez 2007: 33 y ss.).

Dentro de la estrategia empresarial es necesario analizar la implicación de los costes de la calidad, así como sus componentes y bajo las necesidades informativas actuales, la definición de las actividades que general calidad.

Atendiendo a las consideraciones anteriores, en la determinación de las actividades para la evaluación de la política de Calidad Total, debemos considerar un conjunto de variables internas y externas a la organización, pero todas ellas interrelacionadas (Jiménez 1997: 177):

- El entorno donde actúa la empresa

- Un ambiente adecuado dentro de la propia organización

- El grupo directivo encargado de la gestión y dirección 
- El grupo de trabajadores

- Los elementos materiales disponibles y el proceso de producción empleado

- Política destinada a la competitividad

- Política de I+D

- El grupo de proveedores

- Los clientes de la empresa

Estas variables nos condicionan el entorno que influye en la toma de decisiones de gestión de las empresas modernas, considerando que una decisión es una elección consciente entre diversas alternativas analizadas a la que sucede la acción para poner en práctica la alternativa elegida (Ivancevich, Lorenzi, Skinner, Crosby 1995: 158) y que el proceso de toma de decisiones es una serie o concatenación de pasos relacionados que llevan a una decisión, su implementación y su seguimiento. Por lo tanto, la toma de decisiones en el contexto de la gestión de la calidad total se va a fundamentar en tres elementos básicos:

- mejora continua

- gestión basada en hechos

- trabajo en equipo

Los pilares fundamentales donde se sustentan se concretan en los siguientes:

- liderazgo para su consecución

- estrategias y políticas adecuadas

- participación del personal

- orientación al cliente

Estos elementos se pueden identificar con el diagrama de Shewart, también conocido como "ciclo Deming" (ya que fue este autor el que decidió en implantarlo en las empresas americanas), basado en planear, hacer, comprobar y actuar. Las decisiones adoptadas por el grupo se traducen en cambios constantes e incrementales para mejorar el rendimiento de la organización y la satisfacción del cliente. De aquí se deriva la implicación del análisis de los costes de la calidad y su gestión estratégica.

Es dentro de la gestión basada en hechos donde se incorpora el cálculo y la gestión estratégica de los costes de calidad. El modelo de 
costes basado en actividades $(A B C)$ nos va a suministrar información más exacta sobre los costes, que generan valor para la calidad, puesto que estas variables se asocian con las actividades que forman parte del proceso de producción en lugar de hacerlo con el producto final. Con ello podremos lograr la excelencia en la gestión empresarial.

\section{LAS ACTIVIDADES DEL CAMBIO HACIA LA EXCELENCIA}

La filosofía de la RSC o también denominada Responsabilidad Social Empresarial RSE ha supuesto cambios profundos en las organizaciones $y$, así mismo, de los equipos directivos, puesto que han de enfrentarse a cambios profundos en el sistema empresarial, influenciados por el exterior, que a su vez deben considerase como la respuesta o anticipaciones a evoluciones futuras.

Uno de los cambios más relevantes pueden ser la modificación de las circunstancias de la industria derivadas de causas externas e internas, como pueden ser la aparición de un fuerte competidor, la desaparición de clientela importante, la llegada de tecnología revolucionaria a nivel de costes empresariales, llegada de productos sustitutivos, retracción de la demanda, etc. Estas consideraciones implican la necesidad de cambios relevantes.

Una de las actuaciones fundamentales que permiten la amortización de pérdidas derivadas de las circunstancias expuestas anteriormente es la aplicación de la política de la Calidad Total, dentro del marco organizacional, considerada como una de las actividades más relevantes en gestión. A su vez, esta actividad se encuentra relacionada con otras tres actividades que podemos denominar como:

- "hacer mal las funciones necesarias para el proceso productivo",

- "hacer bien determinadas tareas innecesarias",

- "hacer mal las tareas innecesarias", es decir, además de soportar un conjunto de costes no necesarios para la obtención de un producto, la aplicación de factores al proceso de forma ineficiente incrementará de mayor forma los costes soportados, siendo más difícil la reducción en los mismos, puesto que irá acompañado de un estudio de las diferentes funciones, desde un punto de vista técnico y económico, para descubrir los fallos en el sistema empresa y conseguir la mayor eficiencia, tanto técnica como económica. 
Ello nos lleva, a la definición del mapa de actividades de la calidad. Dentro de éste, podemos deducir, que en un principio encontramos dos grandes bloques de actividades, que nos permiten determinar el primer estadio en el mapa de actividades de la Calidad Total.

- De prevención, destinadas a evitar fallos, intentando por lo tanto un mayor esfuerzo a la hora de la realización de la producción, y los de evaluación los procedentes de las pruebas realizadas, inspección y examen, para verificar si se está manteniendo la calidad determinada.

- Con las actividades de evaluación, se pretende detectar los fallos cuanto antes y evitar que éstos puedan llegar a los clientes.

\subsection{Actividades de prevención}

En las actividades de prevención de la calidad, se consumirán factores de costes de prevención. En estas actividades, las tareas que consumen los diferentes tipos de factores se pueden clasificar de la siguiente manera:

Formación: de Recursos Humanos

- Investigación de mercados: Estarían dentro de este grupo, aquellas actividades destinadas al conocimiento de las necesidades, gustos y elecciones del grupo de clientes para adecuar la producción a la información obtenida. Para la realización de la investigación del cliente se realizan los siguientes pasos (Deming 1989: 125):

- diseño del producto

- fabricación; ensayarlo en la línea de producción y en el laboratorio

- ponerlo en el mercado

- ensayarlo en la post-venta; descubrir qué piensa el usuario de él y por qué no lo ha comprado el no usuario.

Revisión: previa al lanzamiento del producto al mercado. Dentro de estas tareas podemos identificar los siguientes tipos:

- Mantenimiento: actividades de puesta a punto de todas las instalaciones y maquinaria (ASQC 1986: 42). 
- Propio departamento de calidad: actividad considerada como el entrenamiento para la calidad y la confiabilidad.

- Planificación de calidad del proveedor y del vendedor: se trataría de reducir, en la medida de lo posible, todos los fallos que se le pueden imputar al proveedor como consecuencia del incumplimiento de la fecha de entrega de los productos, de la entrega del material en las condiciones no establecidas del contrato de compra, entre otros.

- Las auditorias de calidad: Este tipo de auditorias se pueden realizar dentro del ámbito interno de la empresa, bien por el personal encargado de la gestión de la calidad o por auditores independientes, o bien, de carácter externo, sobre los vendedores y el entorno dentro del cual se encuentra insertada la empresa, realizada también por personal interno o por auditores independientes, para analizar el cumplimiento de las normas sobre la calidad, que aunque no son de carácter obligatorio, como las ISO 9000, si son de aconsejable cumplimiento.

- Ingeniería de la calidad: preparación de manuales de calidad y planes de calidad relacionados con los productos o servicios, así como determinadas normas de calidad que considere la empresa de necesario cumplimiento.

- Investigación y Desarrollo: de procesos productivos y tecnologías que supongan un incremento y mejora en las condiciones tecnológicas de toda la empresa.

\begin{tabular}{|l|}
\hline \multicolumn{1}{|c|}{ DISEÑO } \\
\hline - Revisiones de los progreso de diseño de la calidad \\
- Actividades de apoyo de diseño \\
- Ensayo de homologación del diseño del producto \\
- Diseño del servicio-homologación \\
- Pruebas de servicio \\
\hline \multicolumn{1}{c|}{ APROVISIONAMIENTO } \\
\hline - Revisiones de proveedores \\
- Clasificación de proveedores \\
- Revisiones de los datos técnicos de pedidos \\
- Planificación de la calidad de proveedores \\
\hline \multicolumn{1}{c|}{ FABRICACIÓN O SERVICIO } \\
\hline - Procesos de validación de proveedores \\
- Planificación de calidad de operaciones \\
- Planificación de calidad del apoyo a operaciones \\
\hline
\end{tabular}




\subsection{Actividades de evaluación}

En estas actividades se consumen los costes de calidad de evaluación. Estos son los procedentes de la realización de determinadas pruebas, actividades de inspección, examen, determinados tipos de tests, para detectar los posibles fallos de la producción antes de que llegue a los clientes. Se producen como consecuencia de la realización de comprobaciones para conocer el nivel de calidad que ofrece la empresa. Esta comprobación se basa en la realización de determinadas actividades entre las que destacan las siguientes: auditorias, inspecciones, homologaciones y revisiones de la calidad, investigación de mercados, continua formación del personal. Dentro de estas actividades podemos definir las siguientes subactividades.

- Pruebas e inspecciones: las actividades de inspección y pruebas se encargarán de conocer el nivel de calidad de los productos o servicios, de los procesos de diseño, fabricación, comercialización o administración, es decir, que abarcará todas las actividades desarrolladas por la empresa, desde la compra y recepción de los inputs, hasta la comercialización de los outputs.

Con las actividades de inspección y pruebas se aplica la política de una minimización de los costes empresariales, para que el producto y la empresa, en sí misma, sea más competitiva.

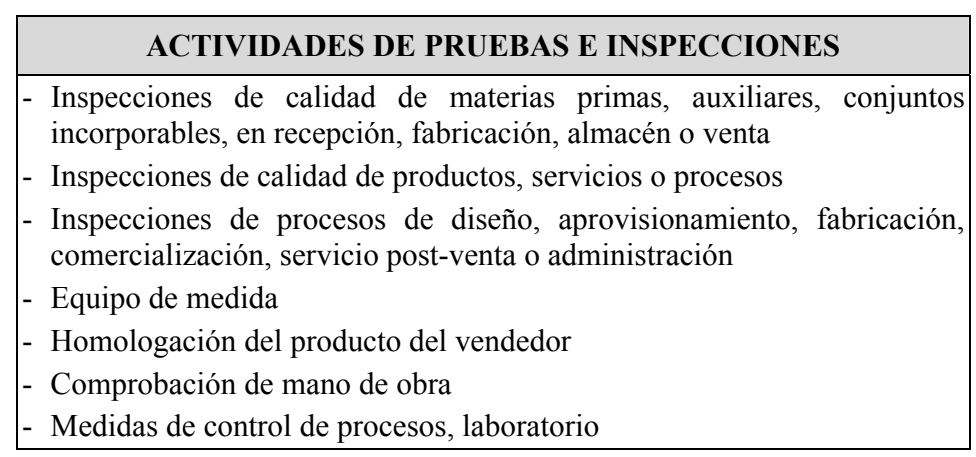

- Investigación de los mercados: actividades de investigación y penetración en los mercados donde hemos lanzado nuestro producto para el conocimiento de su aceptación y su éxito. Se trataría de una investigación destinada fundamentalmente a los clientes, para ver si lo que ellos consideraban como calidad lo han encontrado en el producto 
elegido y en caso contrario, poder conocer los posibles fallos encontrados por ellos, para realizar la correspondiente corrección en aquello que sea necesario, conociendo, al mismos tiempo, la modificación de los gustos y exigencias de los clientes al evolucionar el tiempo y las tendencias de moda.

- Mantenimiento: actividades de reparación y conservación tanto de la instalaciones como de la maquinaria que posee la empresa y que se encuentra afectada al conjunto de sus actividades. La función principal de estas actividades se encaminará al mantenimiento de la capacidad productiva de la empresa y a la obtención de la producción con la misma calidad que cuando la maquinaria se considera nueva y adaptada a las condiciones de calidad planteadas y estimadas por el departamento del Control de Calidad.

- Formación: actividades de Investigación de la calidad, es decir, interpretación y gestión de la documentación y procesos de gestión establecidos, en términos de calidad, incluyendo dentro de este apartado la formación y reciclaje de los altos directivos, encargados de gestionar la unidad económica.

- Investigación y desarrollo: actividades de investigación y puesta en práctica de técnicas de mejora de la productividad y calidad, y por tanto, de la competitividad, una vez realizada la evaluación y el análisis, tanto de la producción obtenida, como de toda la gestión empresarial realizada a lo largo del ejercicio económico, teniendo siempre presente la idea de la excelencia, y mejora continua o Kaizen.

\subsection{Actividades que no generan calidad y no garantizan la}

excelencia

De la clasificación anterior podemos determinar aquel conjunto de actividades que no generan calidad y por tanto que debemos eliminar. De esta forma, compaginaremos la aplicación del $A B C$ con la gestión estratégica de costes.

Para el estudio de estas actividades será necesario la identificación de todas y cada una de la unidades y tareas operativas de la organización para detectar aquello que no contribuye a la consecución de la Calidad Total, intentando cuantificar los factores no sólo fácilmente medibles sino aquellos de carácter cualitativo, pero que se consideran muy importantes, como puede ser el grado de insatisfacción de los clientes. 
destacar:

Dentro de las actividades que no generan calidad podemos

- procesos productivos inadecuados, tanto de la empresa como consecuencia de sus relaciones con los proveedores,

- mala gestión de inventarios, pudiendo solventarse con una política de just-in-time,

- deficiente manejo del servicio de venta y postventa,

- falta de capacitación y formación para la gestión de una política de Calidad Total, incumplimiento de los compromisos con los clientes y,

- carencia de política de investigación de mercados.

La clasificación que se propone de estas actividades es:

\begin{tabular}{|l|}
\hline \multicolumn{1}{c|}{ ACTIVIDADES DE DISEÑO } \\
\hline - Acción correctora del diseño \\
- Reprocesos debidos a cambios de diseño \\
- Desechos debidos a cambios de diseño \\
- Costes de coordinación de producción \\
\hline \multicolumn{1}{c|}{ ACTIVIDADES DE APROVISIONAMIENTO } \\
\hline - Costes de disposición de los materiales adquiridos y rechazados \\
- Costes de sustitución de materiales adquiridos \\
- Acción correctora del proveedor \\
- Reprocesos de los rechazos al proveedor \\
- Pérdida de materiales incontrolados \\
\hline \\
- Revisiones de materiales y acciones correctoras \\
- Disposición de elementos \\
- Análisis de fallos o hacer frente a anomalías de operaciones \\
- Apoyo a la investigación \\
- Acción correctora de operaciones \\
- Pérdida de venta \\
- Fallo en el servicio post venta \\
- Tiempos muertos \\
- Pérdida de imagen y confianza \\
- Desmotivación de Recursos Humanos \\
\hline
\end{tabular}

Independientemente de la clasificación de las actividades descritas, una de las múltiples posibles, en función de las propias características de la organización, en cada una de ellas, a su vez, es posible identificar una serie de subactividades que nos conducirán al cumplimiento del objetivo de la excelencia. Estas son las siguientes: 
- Tormenta de ideas y organización de las mismas. Estrategias a seguir

- Análisis de los procesos y de cada uno de sus componentes

- Análisis de las causas de los problemas y fallos detectados

- Evaluación y análisis de desviaciones entre la planificación y la realidad

- Extracción de información generada y datos mediante la utilización de técnicas estadísticas. Comprobación de resultados

- Conclusión final de la gestión de la calidad total

\section{DRIVERS EN LAS ACTIVIDADES DE GESTIÓN DE LA CALIDAD}

La Gestión de la Calidad Total (TQM) comienza cuando se identifican los clientes de cada persona que trabaja en la organización y definen sus exigencias. Cada función y cada individuo dentro de ella, tienen un conjunto de clientes y cada uno de esos clientes tiene un conjunto de exigencias, ya sean explícitas o latentes. Por ello, el primer paso se centra en la determinación de las exigencias de los consumidores externos, para después identificar las relaciones cliente-proveedor interna y sus exigencias y termina con los proveedores externos. Se conseguirá servir mejor al último cliente que será externo, si cada cliente interno es atendido por el total de la organización en condiciones de oportunidad, completitud y exactitud.

El análisis de actividades de la gestión de la calidad total desarrolla unas funciones específicas como son:

- Definir misiones y responsabilidades de la organización, en su conjunto

- Determinar las necesidades y requisitos de clientes y proveedores

- Definir las tareas que generan valor y no generan calidad, así como definir los diferentes costes de calidad consumidos por las mismas

Para asegurar el éxito de la TQM es necesario relacionar los costes de la calidad con las actividades, puesto que mediante un proceso de identificación de inductores del coste nos permite poner de manifiesto actividades que nacen como resultado de no hacer las cosas de manera correcta.

El enfoque de gestión estratégica de costes, reconoce que el coste es causado o conducido por muchos factores que se encuentran interrelacionados de forma compleja. La comprensión del comportamiento 
de los costes implica comprender la compleja interrelación del conjunto de conductores de costes que funcionan en cualquier situación dada. En el sentido estratégico es necesario explicar el posicionamiento de costes en términos de las elecciones estructurales y capacidades de ejecución que conforman la posición competitiva de la empresa (Mallo y Merlo 2000: 389).

La gestión estratégica implica la utilización de la información sobre costes para el desarrollo e identificación de las estrategias superiores que producirán una ventaja competitiva como la calidad total, que nos va a implicar crear un mejor "valor del cliente". Este concepto puede entenderse como la diferencia entre lo que el cliente recibe y lo que el cliente da por ello.

En la gestión estratégica, existen dos grandes tipos de conductores: estructurales y de ejecución. Entre los primeros encontramos la escala, el ámbito, la experiencia, la tecnología aplicada y la amplitud de la línea de productos a ofrecer a los clientes. Los conductores de ejecución nos permiten analizar la habilidad de la empresa para una gestión estratégica de costes y por tanto, están sometidos a analizar su rendimiento. En esta categoría podemos destacar la participación del capital humano en la consecución del objetivo, la mentalización y la cultura de la dirección, la propia gestión de la calidad total y la explotación de las relaciones con los proveedores y los clientes para la cadena de valor de la empresa.

Con respecto a la gestión de la calidad total, las actividades que hemos clasificado en el punto anterior pueden redefinirse si partimos de la interrelación con la cadena de valor de la empresa. Para potenciar los requisitos o vínculos internos y externos, es necesario identificar las actividades de la empresa y seleccionar aquellas que sirvan para obtener o mantener una ventaja competitiva, como es la calidad total. Dentro de las actividades vinculadas con la Calidad Total se pueden considerar aquellas relacionadas con la organización y con las operaciones de la empresa.

\section{Actividades vinculadas con la organización}

Serán aquellas que consolidan la estructura empresarial a largo plazo y, por lo tanto, sus generadores determinarán la estructura de costes, también, a largo plazo. Afianzan la política de la Calidad Total tanto a nivel estructural como a nivel de procedimientos. Dentro de estas 
actividades, a su vez, debemos diferenciar entre aquellas destinadas a la prevención y las concretas de evaluación.

La relación de actividades y sus correspondientes generadores son la siguiente:

A) Actividades de prevención:

\begin{tabular}{|l|l|}
\hline \multicolumn{1}{|c|}{ ACTIVIDADES ESTRUCTURALES } & \multicolumn{1}{c|}{ CONDUCTORES } \\
\hline - Formación & - Número de personas \\
- Mantenimiento & - Elementos del activo fijo vinculado \\
- Auditorias de calidad & $\begin{array}{l}\text { Cantidad de unidades de trabajo, estilo y filosofía de } \\
\text { la dirección }\end{array}$ \\
\hline
\end{tabular}

\begin{tabular}{|l|l|}
\hline ACTIVIDADES DE PROCEDIMIENTO & \multicolumn{1}{|c|}{ CONDUCTORES } \\
\hline - Investigación de mercados & - Tipos de productos o líneas de productos \\
- Revisión & - Número de departamentos \\
- Planificación de calidad del proveedor y & - Número de proveedores y vendedores \\
del vendedor & - Número de manuales de procedimientos \\
- Auditorias de calidad & - Número de productos, fases del proceso \\
- Ingeniería de calidad & productivo \\
- Prevención medioambiental & - Responsabilidad social \\
- Investigación y desarrollo & - Número de procesos productivos, tipos de \\
& tecnologías de proceso, configuración del producto \\
\hline
\end{tabular}

B) Actividades de evaluación:

\begin{tabular}{|l|l|}
\hline ACTIVIDADES ESTRUCTURALES & \multicolumn{1}{c|}{ CONDUCTORES } \\
\hline - Formación & - Número de personas \\
- Mantenimiento & - Elementos del activo fijo vinculado \\
\hline
\end{tabular}

\begin{tabular}{|l|l|}
\hline ACTIVIDADES DE PROCEDIMIENTO & \multicolumn{1}{|c|}{ CONDUCTORES } \\
\hline - Prevención medioambiental & - Responsabilidad social \\
- Investigación y desarrollo & - Número de procesos productivos, tipos de \\
- Pruebas e inspecciones & tecnologías de proceso, configuración del producto \\
& - Número de procesos \\
\hline
\end{tabular}

\section{Actividades operacionales}

Son aquellas que se desarrollan como consecuencia de la estructura y los diferentes procesos que se han seleccionado para alcanzar la Calidad Total. Dentro de estas actividades podemos diferenciar, según 
la metodología del modelo $\mathrm{ABC}$, aquellas desarrolladas a nivel unitario, a nivel de lote o a nivel de producto. El mapa de actividades y sus correspondientes conductores son:

\begin{tabular}{|l|l|}
\hline \multicolumn{1}{|c|}{ ACTIVIDADES OPERACIONALES } & \multicolumn{1}{|c|}{ GENERADORES } \\
\hline - Pruebas e inspecciones & - Números de productos y servicios \\
- Revisión & - Número de productos; fases del proceso de \\
- Mantenimiento & - Elementos del activo fijo vinculado \\
- Fabricación & $-\mathrm{H} / \mathrm{h} ; \mathrm{h} / \mathrm{máquina}$; $\mathrm{km} / \mathrm{mm} . \mathrm{pp}$, etc. \\
- Rechazos de vendedores & - Número de productos que fallan \\
- Investigación de fallos detectados & - Número de productos rechazados \\
- Actividades de publicidad & - Número de líneas de productos, tipos de mercado \\
- Programación & - Número de unidades terminadas \\
\hline
\end{tabular}

Los diferentes inductores definidos para cada una de las distintas actividades se pueden valorar considerando la metodología de los costes de calidad y sus clasificaciones, que no serán tratados en este trabajo.

\section{CONCLUSIONES}

Bajo la consideración de una gestión estratégica de la calidad, el logro de la excelencia, en base a un posicionamiento de liderazgo en costes, el comportamiento de la dirección de la empresa se debe centrar en los siguientes puntos:

- Considerar a la Calidad Total como la principal estrategia competitiva

- Definir, identificar y analizar los diferentes costes ligados a la calidad

- Elaborar el mapa de las actividades ligadas a alcanzar y cumplir la TQM, así como el conjunto de tareas relacionadas con las mismas, según la estructura anteriormente definida

- Identificar las actividades ligadas a la cadena de valor; éstas son las de prevención y de evaluación. Nos permitirán identificar las actividades que no generan valor $y$ que originan la diferente tipología de los costes de fallos

- Definir los conductores

- Asignar los conductores a las actividades, para obtener el coste total de cada una de ellas 
- Identificación del ciclo de vida del producto final para el cual estamos analizando la gestión estratégica. Consideramos que el ciclo de vida es el tiempo que existe desde su concepción hasta su abandono

Este ciclo de vida está íntimamente ligado con la Calidad Total. Las distintas fases del ciclo de vida son introducción, crecimiento, madurez y liquidación, las cuales intervienen las actividades identificadas anteriormente en la TQM:

- Etapa de introducción: actividades de producción, preparación del pedido, investigación de mercados y lanzamiento, formación del personal encargado del producto en todas las fases, revisión previa al lanzamiento del producto e I+D.

- Etapa de crecimiento: actividades de mantenimiento, del departamento de calidad, protección medioambiental.

- Etapa de madurez: investigación de fallos detectados.

- Etapa de declinación: análisis de rechazos de vendedores, pruebas e inspecciones, investigación de los fallos detectados, servicio postventa en garantía del producto, publicidad y relanzamiento, I+D, apoyo logístico.

- La actividad principal encomendada a un sistema de costes de calidad es la reducción de los costes de fallos. Un ejemplo de control del coste de los fallos es mediante la distribución estadística de Pareto, organizando los distintos fallos, para ser eliminados, comenzando con los elementos de coste más elevados.

- Analizar la necesidad de los costes de evaluación soportados en la organización. Es necesario contestar cuestiones como ¿el programa de evaluación existente origina unos costes demasiado elevados?, ¿se toma como base para realizar actividades de evaluación los niveles más altos alcanzados de calidad?. De esta forma, el análisis de los costes de calidad se emplea para analizar si las actividades de evaluación no son un sustituto de las actividades de prevención adecuadas.

Por ello, la aplicación y el análisis de las actividades para la Gestión continua de la calidad supone un estilo nuevo de dirección, basado en unos principios diferentes a los considerados en el estilo 
tradicional, y centrados en las ideas fundamentales del "Corporate Governance". Entre estos principios podemos destacar:

a) Participación

b) Discusión abierta y continua

c) Seguridad en el puesto directivo y de trabajo

d) Mejora continua y excelencia en todos los aspectos y variables empresariales

e) Política de Recursos Humanos centrados en la culturización sobre la mejora continua

f) Relaciones con proveedores y clientes

g) Acercamiento a las personas

h) Formación continuada en base a alcanzar la excelencia

i) Nueva cultura empresarial basada en la calidad

j) Identificación de objetivos y no conflicto de objetivos personales y corporativos

En definitiva, la aplicación de esta metodología nos permitirá contestarnos a cuatro preguntas básicas, que nos servirán como autocontrol en la gestión desarrollada. Estas cuestiones son ¿Qué cultura empresarial seguimos y quienes somos?, ¿Qué actuaciones estamos desarrollando en este entorno de excelencia?, ¿Conocemos a nuestros clientes y sus demandas, presentes y futuras? ¿La política seguida con los proveedores es la acertada en la generación de valor global? ¿Se han cumplido los objetivos establecidos y planificados? ¿Cuál es el camino a seguir para el futuro?

\section{BIBLIOGRAFÍA}

ASQC (1986) I costi della qualita: definizione, controllo e riduzione. Torino: ISEDI.

BRIMSON, J.A. (1991) Activity Accounting. An Activity-Based Costing Aprroach. New York: John Wiley and Sons Inc.

CROSBY, P.B. (1979) Quality is Free. New York: McGraw-Hill.

DemING, W.E. (1989) Calidad, Productividad y Competitividad. La salida de la crisis. Madrid: Ed. Diaz de Santos, S.A. 
FUNDACIÓN ENTORNO: "El papel de la Empresa en la Sociedad". Www. fundacionentorno.org.

HARRINGTON, H.J. (1991) El coste de la mala calidad. Buenos Aires: Ed. Díaz de Santos, S.A.

HOWE, R.J.; D. GAEDDERT and M.A. HOWE (1993) Quality on Trial: Bringing Bottom-line Accountability to the Quality Effort. New York: McGraw Hill Book Company.

IMAI, M. (1986) Kaizen: The Key to Japan's Competitive Success. New York: Random House.

ISHIKAWA, K. (1994) Introducción al control de la calidad. Madrid: Díaz de Santos.

IVANCECICH, J.M.; P. LORENZI, S.J. SKINNER, P.B. CROSBY (1995) Gestión, calidad competitividad. Madrid: Ed. Irwin.

JIMENEZ, M.A. (1996) La Calidad como estrategia competitiva. Gestión, rentabilidad y auditoria. Madrid: Ed. Tébar Flores.

- (1997) Los costes de la calidad en la Contabilidad de Gestión. Madrid: Instituto de Contabilidad y Auditoría de Cuentas. Ministerio de Economía y Hacienda.

JURAN, J.M. (1974) Quality Control Handbook, tercera edición. New York: McGraw-Hill, Inc.

- (1990) Juran y el liderazgo para la calidad. Madrid: Ed. Diaz de Santos, S.A.

LEAVITT, J.S. (1994) Total Quality Project Management. New York: McGraw Hill, Inc.

LIZCANO, J.L. (2006) "Buen gobierno y responsabilidad social corporativa", Partida Doble, $\mathrm{n}^{\circ} 182$, pp. 21-35.

LORENZO DE MEBIELA, J.B. (2007) "Etica y gestión ética: nuevos retos gerenciales", Capital Humano, n² 213, Septiembre, pp. 106-113.

Mallo, C. (1988) Contabilidad de Costes y de Gestión. Madrid: Ed. Pirámide, S.A.

- y M.A. Jimenez (2007) Contabilidad de Costes. Madrid: Ed. Pirámide, S.A.

-; R.S. Kaplan, D. MelJem, C. Gimenez (2000) Contabilidad de costos y estratégica de gestión. Madrid: Prentice Hall. Pearson Educación, S.A.

OCDE (2004) Principios de Gobierno Corporativo.

Pedersen, W. (1962) Los costes y la política de precios. Madrid: Ed. Aguilar. 
PORTER, M.E. (1999) Ser Competitivo. Bilbao: Ed. Deusto, S.A.

QUESADA, F.J.; M.A. JIMENEZ, J. GARCIA (2001) Sistemas Informativos Contables para el análisis empresarial. Una visión integrada para la valoración de empresas. Madrid: Prentice Hall, colección Financial Times.

- y - (2008) "Aprendizaje y liderazgo en la responsabilidad social de las empresas", Revue Sciences de Gestion, n 66, pp. 153-174. 